J3eA, Journal sur l'enseignement des sciences et technologies de l'information et des systèmes, Volume 4, Hors-Série 2, 25 (2005)

DOI : http://dx.doi.org/10.1051/bib-j3ea:2005725

(C) EDP Sciences, 2005

\title{
Introduction aux systèmes pile à combustible
}

D. Hissel, D. Bouquain et A. Miraoui

Département Génie des Systèmes de Commande (GSC)

Université de Technologie de Belfort-Montbéliard (UTBM)

rue Thierry Mieg

F-90010 Belfort CEDEX, France 


\title{
INTRODUCTION AUX SYSTEMES PILE A COMBUSTIBLE
}

\author{
Daniel HISSEL, David BOUQUAIN, Abdellatif MIRAOUI \\ Département Génie des Systèmes de Commande (GSC) \\ Université de Technologie de Belfort-Montbéliard (UTBM) \\ Rue Thierry Mieg, 90010 Belfort Cedex \\ daniel.hissel@utbm.fr
}

\begin{abstract}
Résumé :
Cet article décrit un système didactique original à destination des élèves ingénieurs du département Génie des Systèmes de Commande (GSC) de l'UTBM. L'objectif pédagogique général est de se familiariser avec le fonctionnement d'un système pile à combustible et de mettre en évidence les principales contraintes inhérentes à celui-ci. De plus, une illustration des verrous technologiques à surmonter avant l'apparition sur le marché de véhicules à pile à combustible sera réalisée au travers d'un petit véhicule de démonstration.
\end{abstract}

Mots clés : énergies alternatives, pile à combustible PEMFC, énergie solaire.

\section{Introduction}

Depuis le début des années 90, les recherches sur les Piles à Combustible (PAC) connaissent une croissance fulgurante. Ceci est dû à une conjonction de phénomènes. D'une part, les réserves de pétrole, bien que difficilement quantifiables sont vouées à l'épuisement [1]. D'autre part, la pollution atmosphérique devient chaque année plus préoccupante, les moyens de transport y contribuant pour une large part (estimée à près du tiers aux Etats-Unis) [2]. L'idée d'un véhicule tout électrique est également séduisante au niveau des émissions sonores ainsi qu'en ce qui concerne le rendement potentiellement élevé de la conversion électrochimique. Ainsi, de nombreux démonstrateurs ont d'ores et déjà été proposés par tous les grands constructeurs automobiles [3]. Ces véhicules reposent tous sur des chaînes de traction électriques; l'importance de l'EEA dans le domaine de l'automobile s'en trouvant considérablement renforcée. Les élèves ingénieurs du domaine de l'EEA se doivent donc d'être sensibilisés aux contraintes relatives à ces futurs ( ?) standards automobiles; ceci quand bien même il semble encore utopique d'envisager une date de commercialisation.

\section{Description du matériel.}

Afin d'initier ses élèves ingénieurs au système pile à combustible, le département GSC a fait l'acquisition d'un banc d'essai d'une pile à combustible de type PEMFC (pile à membrane échangeuse de protons). Ce banc est par ailleurs complété par un petit véhicule électrique à pile à combustible permettant de mettre en évidence la chaîne énergétique mise en jeu en allant de la production d'hydrogène à son utilisation en passant par un stockage énergétique intermédiaire.

\section{a) Petit véhicule électrique.}

Ce véhicule de faible puissance (environ $0,1 \mathrm{~W}$ ) est utilisé en début de manipulation afin d'illustrer le principe général de la conversion d'énergie (cf. Fig. 1). Ici, il s'agit de mettre en évidence la chaîne de conversion énergétique suivante : énergie solaire $\rightarrow$ énergie électrique $\rightarrow$ production d'hydrogène et d'oxygène $\rightarrow$ stockage conversion électrochimique $(P A C) \rightarrow$ génération d'électricité $\rightarrow$ alimentation $d u$ moteur d'entraînement.

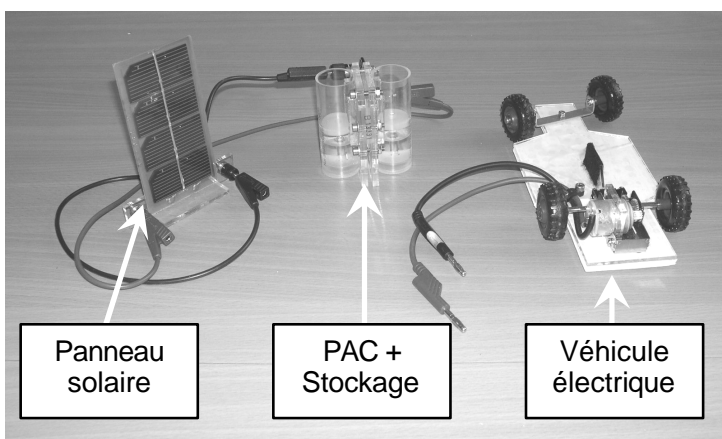

Fig. 1 : Petit véhicule électrique à PAC.

La manipulation est constituée d'un panneau solaire d'une surface de $40 \mathrm{~cm}^{2}$, d'une cellule élémentaire de pile à combustible de type PEMFC accompagnée de deux réservoirs permettant de stocker l'oxygène et l'hydrogène et enfin d'un petit véhicule sur lequel est monté un moteur à courant continu de $0,1 \mathrm{~W}$.

b) Banc d'essai.

Le banc d'essai pile à combustible didactisé (cf. Fig. 2) permet quant à lui de déterminer et d'étudier les différentes caractéristiques d'une pile PEMFC. 


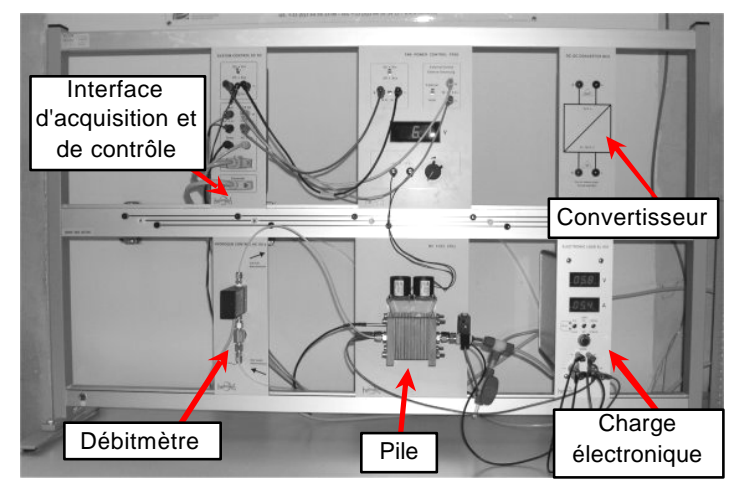

Fig. 2 : Implantation des différents éléments.

Il est composé des éléments suivants :

- Une alimentation en hydrogène issue d'une bouteille d'hydrogène comprimé. Cette dernière est stockée, pour des raisons de sécurité, à l'extérieur du bâtiment dans un abri grillagé dédié à cet effet. L'alimentation est équipée d'un débitmètre massique permettant de déterminer la consommation de combustible.

- Une pile à combustible PEMFC. Sa puissance nominale est de $30 \mathrm{~W}$ pour une puissance maximale de 50W. Sa tension à vide est d'environ 9V. Dans notre cas la pile fonctionne en mode fermé. On alimente donc la pile en hydrogène du côté anode sous une pression de 1.7 bars absolus et la sortie hydrogène de la pile est fermée par une électrovanne. La pile consomme donc uniquement la quantité d'hydrogène nécessaire à la fourniture du courant demandé par la charge.

- Deux ventilateurs alimentent la pile en air mais permettent également le refroidissement de celle-ci. Un système de pilotage permet d'ajuster la vitesse de rotation des ventilateurs.

- Une électrovanne de purge est placée à la sortie d'hydrogène de la pile. En effet, la membrane échangeuse de protons laisse également passer une partie de l'azote contenu dans l'air ainsi que de l'eau qui, à la longue, réduit l'accessibilité de l'hydrogène sur les sites catalytiques. Ce phénomène conduit à une dégradation des performances de la pile. En purgeant à intervalles réguliers le compartiment anodique de la pile, on assure le fonctionnement permanent de celle-ci. Les rejets issus de ces purges sont évacués à l'extérieurde la pièce.

- Une sonde de température mesure la température interne des cellules. Il s'agit d'une sonde PT100 qui traverse de part en part la pile.

- Une charge électronique pilotée en courant ou en puissance fait débiter la pile. Cette charge dispose d'une entrée de consigne externe. Cette interface permet donc de réaliser des essais dynamiques sur la pile.
- Un convertisseur de tension transforme la tension variable issue de la pile (de 2 à $9 \mathrm{~V}$ ) en une tension fixe de 9V. Ainsi il est possible d'alimenter tous les auxiliaires du banc grâce à ce module.

- Une interface d'acquisition et de contrôle est présente, elle a pour rôle de collecter les diverses informations du banc pour les transmettre à un PC. D'autre part le PC pilote aussi les différentes variables du banc via cette interface.

- Un logiciel d'acquisition et de contrôle réalise le traitement des données et le pilotage du banc. Il assure l'affichage et le stockage des différents paramètres : tension PAC, courant PAC, température PAC, débit d'hydrogène ainsi que la tension d'alimentation des ventilateurs. Bien entendu, il assure également un rôle de contrôle en pilotant le courant de la charge électronique, la vitesse de rotation des ventilateurs et la fréquence des purges.

\section{c) Evolutions du banc.}

Après un semestre d'utilisation, le banc a donné entière satisfaction. Néanmoins, afin de préserver l'intégrité de la pile à long terme, le département GSC a développé un nouveau logiciel de contrôle du banc intégrant différentes sécurités pardonnant ainsi les erreurs de manipulation (cf. Fig. 3). Ce logiciel a été réalisé à partir de l'interface Labview. Il intègre différentes sécurités telle que par exemple la protection en température de la pile : si la température atteint le seuil maximal supporté par la pile $\left(50{ }^{\circ} \mathrm{C}\right.$ dans notre cas), la consigne de courant de la charge électronique est inhibée et la pile est ventilée au maximum. L'utilisateur peut "reprendre la main" uniquement lorsque la température tombe en dessous de $45^{\circ} \mathrm{C}$. De même le logiciel intègre le contrôle et la protection en tension de la pile.

D'autre part il permet de stocker les mesures dans un tableau à intervalle ajustable afin de faciliter le post-traitement des données par d'autres logiciels tels que Matlab ou Excel.

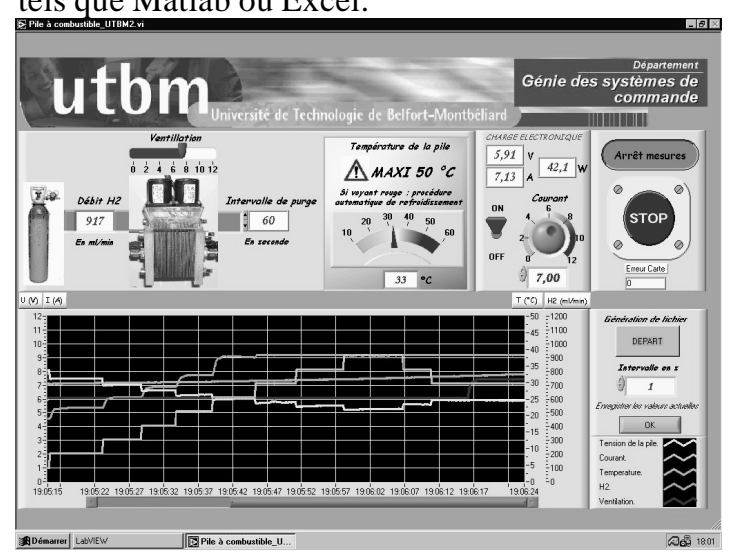

Fig. 3 : Logiciel d'acquisition et de contrôle.

Une évolution du logiciel permettra prochainement de réguler la vitesse des ventilateurs en fonction du 
courant débité, tout en évitant l'assèchement des membranes. Ce point est particulièrement délicat à mettre en œuvre. En effet, si le flux d'air est trop important, il y a risque d'assécher la membrane; si le flux d'air est trop faible, la température de la pile risque de monter et par évaporation, également de conduire à un assèchement de la pile...

Des évolutions du matériel sont encore à venir : nous allons instrumenter la pile à l'aide de micro thermocouples afin d'obtenir une cartographie thermique $3 \mathrm{D}$ de la pile. Des investigations sur l'amélioration du débit d'air et sur le contrôle de l'hygrométrie sont également en cours. En effet, comme la ventilation agit sur trois paramètres qui sont la quantité d'air transitant dans la pile, la température de la pile et l'humidification, il parait judicieux de pouvoir affiner cette variable en fonction du point de fonctionnement désiré.

\section{Manipulation et résultats expérimentaux.}

\subsection{Principes d'utilisation des PAC dans l'automobile}

La première partie de la manipulation proposée aux étudiants est destinée à se familiariser à l'utilisation des PAC dans l'automobile. Il s'agit ici (sur la base du petit véhicule électrique décrit précédemment) de suivre la filière hydrogène de sa production à son utilisation embarquée.

a) Rendement du panneau solaire.

On connecte tout d'abord le panneau solaire, éclairé par une lampe halogène, à la PAC afin de faire l'électrolyse de l'eau. Un luxmètre est utilisé pour mesurer l'éclairement (noté E) émis sur le panneau solaire. La puissance reçue par le panneau est donnée par:

$$
P=\frac{E . S}{K}
$$

avec: $\quad S$ : surface du panneau solaire $\left(\mathrm{m}^{2}\right)$

$K$ : efficacité lumineuse $(K=20 \mathrm{~lm} / \mathrm{W}$ pour une lampe halogène)

Dans notre cas, nous relevons une puissance reçue de $1.3 \mathrm{~W}$ en appliquant cette formule. Il suffit alors de mesurer la puissance électrique délivrée par le panneau solaire (dans notre cas $\mathrm{P}_{\mathrm{PS}}=108 \mathrm{~mW}$ ) pour évaluer le rendement du panneau solaire considéré (très faible ici, de l'ordre de $8 \%$ ).

b) Alimentation du véhicule.

A partir de la mesure de la quantité d'hydrogène produite par la réaction d'électrolyse de l'eau en $1 / 2$ heure $(14 \mathrm{ml})$, il s'agit ici d'évaluer le temps de fonctionnement du véhicule. On supposera que l'intégralité des $14 \mathrm{ml}$ d'hydrogène présents peut être consommée.
Comme notre PAC n'est composée que d'un seul élément, la relation liant la quantité d'hydrogène consommé au courant délivré par la pile est:

$$
q_{H_{2}}=\frac{I}{2 F}
$$

avec : I : courant délivré par la pile F : constante de Faraday $\mathrm{q}_{\mathrm{H} 2}$ : débit molaire

En fonctionnement, on mesure un courant moyen $130 \mathrm{~mA}$, ce qui conduit à une consommation de $6,7.10^{-7}$ mol.s ${ }^{-1}$ d'hydrogène. Pour un volume stocké de $14 \mathrm{ml}$, nous parvenons à une durée de fonctionnement voisine de 14 minutes.

c) Stockage d'hydrogène.

Il serait bien entendu indispensable de supprimer l'étape de stockage préalable d'hydrogène à bord du véhicule, celle-ci étant relativement longue dans notre cas de figure [4]. De plus, à grande échelle, une telle chaîne énergétique ne semble pas très réaliste. Le stockage d'hydrogène devrait, à plus grande échelle, être centralisé en un point fixe. Ceci impliquera cependant la présence de tout un réseau de distribution d'hydrogène, lequel est à ce jour inexistant.

\subsection{Caractérisation d'un générateur PAC de $50 \mathrm{~W}$}

Dans une deuxième partie de la manipulation, les étudiants s'intéressent plus particulièrement au générateur $\mathrm{PAC}$ de $50 \mathrm{~W}$ et à sa caractérisation statique et dynamique.

\section{a) Caractéristiques statiques.}

Cette partie est consacrée au relevé des caractéristiques statique $\mathrm{U}=\mathrm{f}(\mathrm{I})$ et $\mathrm{P}=\mathrm{f}(\mathrm{I}) \quad \mathrm{du}$ générateur électrochimique qu'est la pile à combustible. On cherchera également à quantifier l'influence du débit d'air cathodique sur ces caractéristiques.

Après cette première étape, les étudiants sont invités à évaluer le rendement électrique du stack définit comme étant le produit :

- d'un rendement en tension :

$$
\eta_{U}(I)=\frac{U(I)}{U_{t h}}
$$

avec $U_{t h}=1.25 \mathrm{~V} /$ cellule élémentaire

- et d'un rendement en courant :

$$
\begin{gathered}
\eta_{I}(I)=\frac{I}{I_{t h}} \\
\circ \quad \text { avec } I_{t h}=\frac{\dot{V} \cdot F \cdot z}{V_{m} \cdot n} \\
\left.\circ \quad \dot{V} \text { : débit volumique d'hydrogène (en } 1 . \mathrm{s}^{-1}\right)
\end{gathered}
$$


- F est la constante de Faraday

- $z$ est le nombre d'électrons échangés par la réaction électrochimique

- $n$ est le nombre de cellules élémentaires dans le stack

- $V_{m}$ est le volume molaire de l'hydrogène à $0^{\circ} \mathrm{C}$ et $10^{5} \mathrm{~Pa}\left(\right.$ en $\left.1 . \mathrm{moI}^{1}\right)$

L'évolution typique de ce rendement électrique est donnée sur la figure 4 .

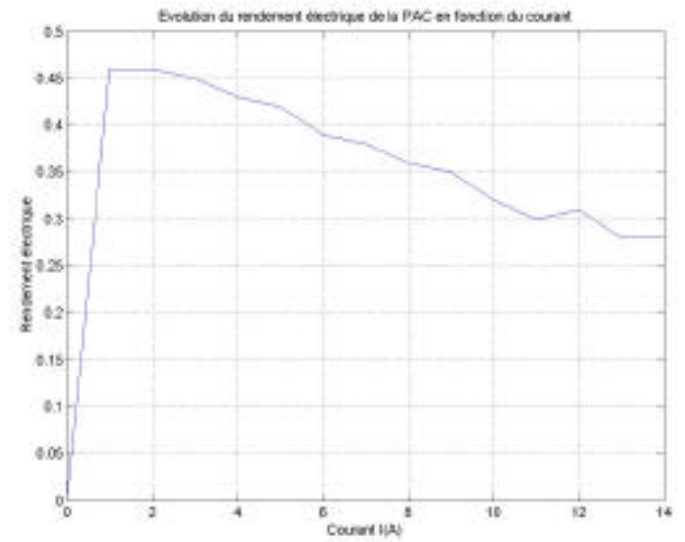

Fig. 4 : Rendement électrique de la PAC.

b) Fonctionnement en mode dynamique.

D'autres essais sont consacrés à l'étude du comportement dynamique de la pile à combustible. Un débit d'air est fixé sur les ventilateurs et des purges sont effectuées à intervalles de temps constants sur le compartiment anodique de la PAC. Un cycle de courant est imposé par la charge électronique sur la pile (Fig. 5).

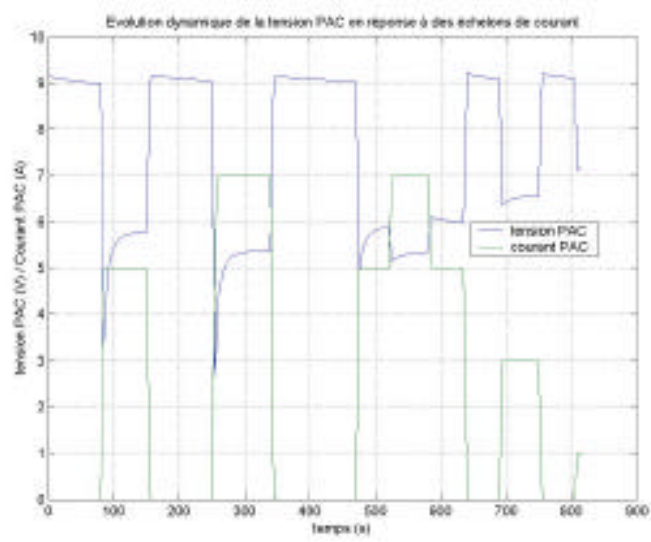

Fig. 5 : Essais dynamiques de la PAC.

On peut constater que la PAC répond instantanément en courant, néanmoins la tension va mettre un certain temps à se stabiliser. Le temps de réponse de la tension est de l'ordre de 50 secondes. Un tel temps de réponse semble difficilement compatible avec des exigences importantes de dynamique. Ceci nous amène naturellement à envisager une hybridation de la PAC avec des dispositifs de stockage énergétique pour des applications véhicule.

\section{Conclusion}

L'objectif de la manipulation est de proposer aux étudiants du département GSC de l'UTBM une première prise de contact avec les générateurs pile à combustible. L'idée générale est de clarifier leur utilisation dans des véhicules de transport et de mettre en évidence les principaux points délicats qui restent encore mal maîtrisés (fonctionnement à rendement maximal, fonctionnement en mode fermé, fonctionnement dynamique, hydratation de la membrane, ...). Comme son nom l'indique, cette manipulation ne constitue cependant qu'une introduction aux PACs, d'autres manipulations mettant en exergue tel ou tel point particulier seront proposées dans un avenir proche.

Dans un autre registre, ce banc d'essai, de par son caractère complètement ouvert, va également permettre de réaliser des travaux pratiques d'automatique (par exemple asservissement de température du stack, asservissement de débit d'air). Au niveau informatique industrielle, ce système didactique est également une excellente plate-forme pédagogique ; en effet, il est possible de faire évoluer le système d'acquisition et de contrôle (base Labview) et de réaliser un dialogue entre les différents sous systèmes par un bus de terrain.

Finalement, il est également envisagé de coupler ce système didactique à des dispositifs de stockage d'énergie électrique (batterie ou supercondensateurs) pour alimenter de petits véhicules. L'objectif recherché sera alors la détermination et la réalisation de convertisseurs statiques dédiés et la gestion d'énergie sur un système hybride.

\section{Bibliographie}

1. Hissel, D., Péra, M.C., François, X., Kauffmann, J.M., Baurens, P., "Contribution to the modelling of automotive systems powered by polymer electrolyte fuel cells", EAEC'01 European Automotive Engineers Congress, pp. A-319-A-326, Bratislava, Slovaquie, 2001.

2. Péra, M.C., Hissel, D., Kauffmann, J.M., "Fuel Cell Systems for Electrical Vehicles: an Overview”, IEEE Vehicular Technology News, vol. 49, n 1 , pp. 9-14, 2002.

3. Corgier, D., "Le programme de demonstration de bus à pile à combustible d'Irisbus", Colloque Energie Electrique et Transports Terrestres, pp. 5258, Belfort, 2003.

4. Beretta, J., "Stockage d'énergie dans les transports terrestres, actualité et perspectives", Colloque Energie Electrique et Transports Terrestres, pp. 47-51, Belfort, 2003. 